\title{
Multi-agent Based Protection on Highly Dominated Distributed Energy Resources
}

\author{
Stephanus A. Ananda, Jyh-Cherng Gu \\ Department of Electrical Engineering, National Taiwan University of Science and Technology. \\ Email: d10007801@mail.ntust.edu.tw,jcgu@ee.ntust.edu.tw \\ Received January, 2013
}

\begin{abstract}
Highly penetration of Distributed Energy Resources (DER) on the grid systems nowadays makes the systems grow dynamically. The system become more complex and the protection system become more complicated. The protection relay should accommodate the system changes according to the system conditions and topologies. As part of developmental aspect of Distributed Artificial Intelligent, Multi Agent System (MAS) is a challenging method for improving the intelligent properties of relay protection. This paper introduces the use of MAS approach on radial distribution system protection dominated with DER using dispersed adaptive rule-based protection supported by distributed database agent. The simulation results confirmed that the proposed algorithm can respond within $15.05 \mathrm{~ms}$.
\end{abstract}

Keywords: Multi-Agent System; Power System Protection; Distributed Energy Resources

\section{Introduction}

As the economic growth, the electricity demands are increased and the need of electrical power sources is growing. Along with the awareness of green energy and cost minimization, the energy providers begin to look to new strategy. They use small-scale power generator such as micro turbine, wind turbines, photovoltaic, etc. and placed them near to the load center along with end-user's "share power generator" to expand power sources capacity and creates Distributed Energy Resources (DER) [1]. This DER has the advantage in maintaining system stability, increasing power quality and reliability of the system, reducing the distribution cost, and provides supply in islanding mode [2-4]. The renewable energy DER is also considered can reduce the air pollutant emission [2]. With the DER connected in the system, it will create subsystem called micro-grid.

Beside those all advantages, connecting DER on the radial distribution system can affect the load flow in the system and change the fault current direction and magnitude $[5,6]$. It will mitigate the sensitivity and selectivity of existing relay and cause problems in protection system. Protection coordination becomes more complicated. Many papers investigated the impact of DER installation on existing protection systems. Some of the problems that were reported are false tripping, protection blinding and unsynchronized in the automatic reclosing $[5,7]$. The other problem is the DER that use power electronics (PE) interfaces has current limiter that also limit the fault currents from the DER. Small micro turbine or combine heat and power (CHP) also has limitation on fault current and short period of time to achieve out of step condition [8]. This means relay protection must have different setting for different grid condition. Oudalov [8] proposed adaptive relay protection scheme based on the SCADA system to overcome this problem, meanwhile A.R.Haron, A. Mohamed, and H.Shareef [3] proposed resetting over current relay protection or using microprocessor relay with artificial intelligent approach. Edward Coster, Johanna Myrzik and Wil Kling [5] proposed more modern approach using Multi-Agent System (MAS) to get optimal protection on micro-grid.

\section{Multi Agent System Technology}

\subsection{Multi Agent System Technology}

A summarizing from Stephen D.J.'s literature study [9-11], Agent and Multi Agent concepts are originated from computer science society. Many definitions about agent can be found; it can clearly show the difficulty to get satisfied definition about the agency. But among many different definitions, they share the same basic concepts those are the notion of agent, environment and autonomy properties. According to Wooldridge, an agent is: a software (or hardware) entity that situated in some environment and is able to autonomously react to a change in that environment. Environment is defined as everything outside the agent and at least some part of the environment can be observable and also can be alterable by agent. Observable means agent can take the informa- 
tion either physically from a sensor or by program invocation in the computing environment [9]. Alterable means the agent can change the environment either physically by tripping the circuit breaker (CB) or storing information in database. Wooldridge extend the definition to the agents become with intelligent agent by adding flexible autonomy to the definition. Flexible autonomy means that the agent has three characteristics i.e. 1) Reactivity, the agent ability to react due to the environment changing; 2) Pro-activeness, the agent ability to take initiatives; 3) Social Ability, the agent ability to communicate to other agents.

\subsection{Multi Agent}

Multi agent is a system composed by two or more agents or intelligent agents communicates each other and work synergistically to achieve the system goal, while also at the same time each agent will try attaining the local goal [9]. As part of Distributed Artificial Intelligent (DAI) system, MAS is very attractive to use in distributed power system [12].

\section{Multi-Agent Based Protection}

\subsection{Multi-Agent System Architecture}

Unlike SCADA protection system that based on the centralized operation architecture, MAS has decentralized architecture and creates distributed relay system structure. Each agent will act as intelligent node that in collaborate with other agents via communication infrastructure in order to solve the problem in the power system protection. The agent itself can be a sensing unit, a protection device, a processing unit or database unit [13].

Many different MAS structures, architectures and agents configuration were proposed [12,14-20], but most of them have similarity in hierarchical classification. Generally they divide the structures into 3 layers. First layer is the equipment layer that functions to get the data from and send command to the power system (I/O function). The second layer is substation / coordination layer that functions to coordinate and compute the process data from the first layer. The third layer is system layer that functions to collect and evaluate data.

\subsection{Multi-Agent System in Protection Systems}

In the multi agent-based protection systems, the closest layer to the real equipment in the power systems is the equipment layer. Equipment layer is the layer that usually composes by measurement agent like CT and PT agent, performer agent like $\mathrm{CB}$ agent and protector agent like bus agent, line agent, etc. The information gathered by the measurement agents will be send to protector agent. Protector agent will calculate the fault current and then send the information to substation (coordination) layer. Management agent in substation layer will receive data from equipment layer and determine the fault type and the fault location. Then management agent will identify the problem and finding the type of the solution (or relay setting) should be used. Based on the solution chosen, the management agent will send a command to $\mathrm{CB}$ agent as a performer agent in the equipment layer whether to open or close the $\mathrm{CB}$ contact. At the same time management agent will send to the evaluation agent in the system layer to be evaluated and back up. The evaluation result will be reported to the user for system improvement. Sometimes the evaluation agent can also contribute to improve the problem solution in management agent in the substation (coordination) layer.

The other agents also needed are database agent and communication agent. Database agent usually concentrated in the second layer. Meanwhile, the communication agent should be merging to individual agent to enable every agent to communicate each other's.

\section{Proposed System}

In the highly dominated DER, especially CHP type, the generators have small inertia, so they easily out of step when the short circuit occurs in the systems. The protection should response quickly to anticipate this problem.

To solve this problem, this paper proposes dispersed adaptive rule-based protection supported with distributed database agent. The proposed method is based on Oudalov et al.'s centralized adaptive protection algorithm [8] and P. C. Maiola [20]. The algorithm will be implemented in the protection agent inside the equipment layer supported by local database agent instead of concentrating it on single management agent.

\subsection{Proposed Architecture}

The proposed architecture of MAS based protection can be seen in the Figure 1. In the equipment layer, IED agents are used as combined function of CT agent, PT agent, $\mathrm{CB}$ agent extend with local database agent and local knowledgebase agent that formed by local coordination agent and local configurator agent that acted as distributed database and distributed knowledgebase. Because this agent is used as Intelligent Electronic Device (IED) so it named as IED agent and the structure can be seen in Figure 2. The agents inside IED Agent are named as unit for clarity.

Substation layer are consist of coordination agent and configurator agent. Coordination agent is responsible for identifying fault type and fault location according the information received from IED Agents. Configurator agent is an expert system that decides which solution or relay setting should choose to overcome the problem and 
also provide the back-up system protection scenario. The Configurator agent will support by database agent to store the data i.e. list of rules of the solution, grid topologies, etc.

System layer is consist of evaluation agent, that will evaluate every condition occurs in the system. The agent will calculate the system's condition offline and evaluate if the protection system already running properly and efficiently.

Database Agent is agent that stores all the information calculated by the other agent such as relay settings, configuration / topology changing and also faults history. DF (Directory Facilitator) Agent is agent that has a special function to register the services. AMS (Agent Management Server) is agent that has a special function to register the all agents used in the MAS. Every agent will communicate each other using TCP/IP protocol.

In the real system IED Agent (A01-A12) are installed at the position of the $\mathrm{CB}$ (see Figure 3). Every IED Agent will connect to other agents via TCP/IP communication protocol.

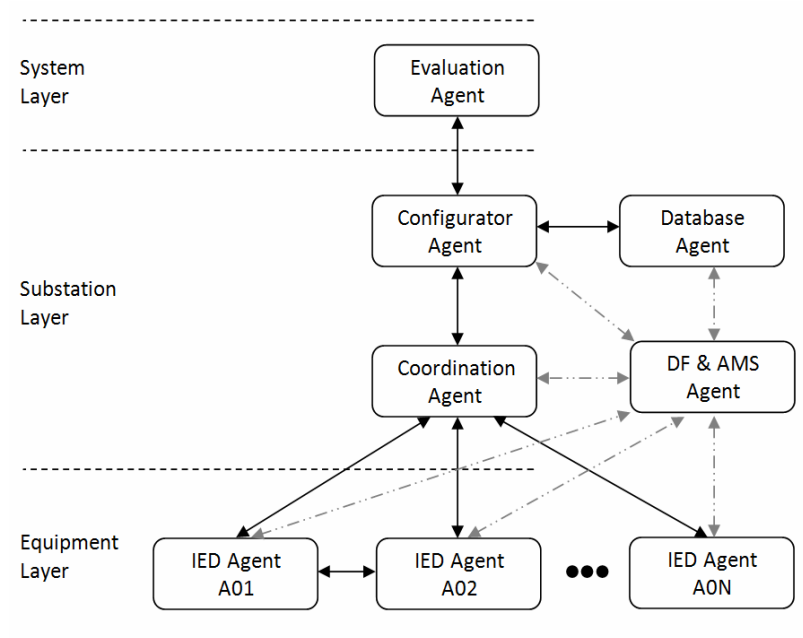

Figure 1. Block diagram of proposed MAS protection architecture.

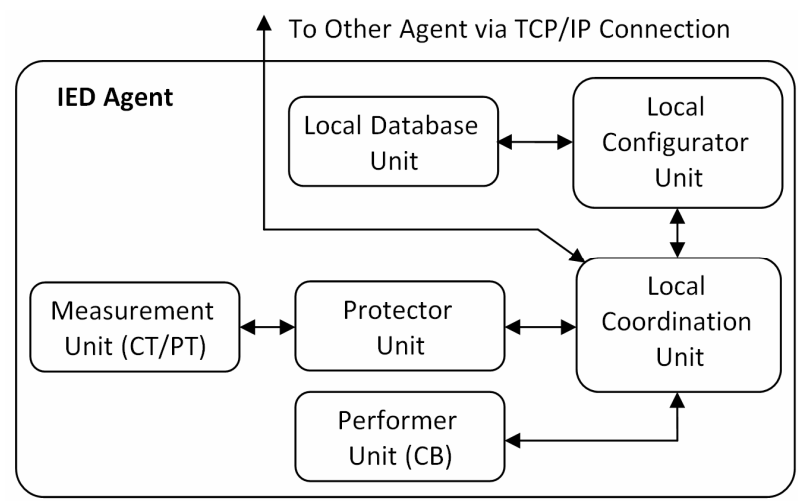

Figure 2. Block diagram of IED agent architecture.

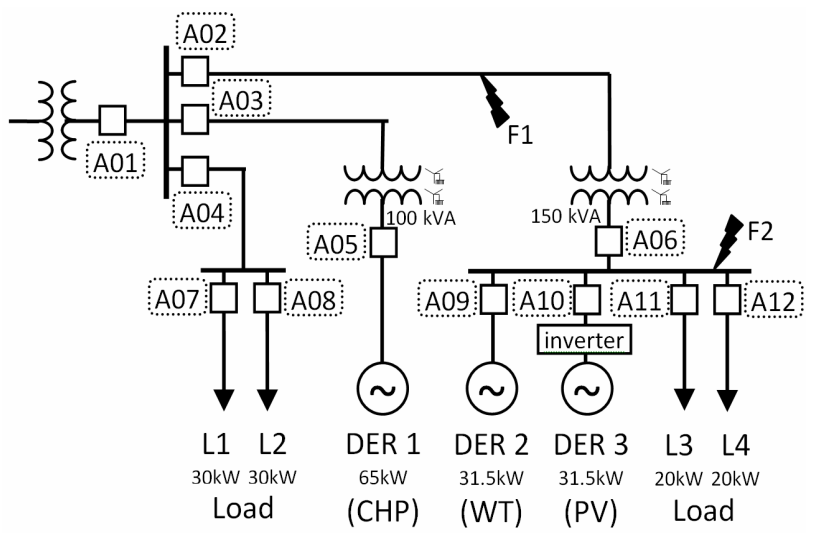

Figure 3. Distribution feeder with DER and MAS protection.

\subsection{Proposed Algorithm}

In the beginning, all agents need to register their name and function to AMS agent and DF agent respectively. Every IED Agent should check and list the neighbor IED Agents which are connected to them. This will perform subsection topology.

After agent initialization finished, IED Agents will start to measure and monitor the system current and voltage every certain time via measurement unit (CT/PT) and send to Protector Unit (see Figure 2). The Protector Unit will calculate and determine the fault current and maximum load current when a changing situation like fault happens in the network. If faults occur Protector Unit will send the information to the Local Coordination Unit.

After receiving information from Protector Unit, Local Coordination Unit will first check the network grid condition is in islanding situation or grid connected by checking the status of the agent at main breaker. Next step, Local Coordination Unit will request information from neighboring IED Agent. From all data gathered Local Coordination Unit will determine and calculating the fault type. All information will send to Local Configuration Unit to get the "Relay Setting Configuration". After determined the relay setting, the Local Configuration Unit will send the information to Local Coordination Unit what condition should be achieved. The Local Coordination Unit will send Trip Signal to CB through Performer Agent. The Local Coordination Unit will send the report information to Coordination Agent of Substation Layer.

In the Substation Layer, Coordination Agent will gather data from every IED Agent in the whole system. Coordination agent will check whether the fault is cleared by IED Agent or not. If it is cleared, the Coordination Agent will capture the new topology and send all information to Evaluation Agent in the System Layer for evaluation. 
If the fault condition still occur, the Coordination Agent will calculate the fault location and send the information to Configuration Agent to get the "Relay Configuration" list, that is the list of which relay will operate and which relay will block according the situation. After received the list, the Coordination Relay will send the information to all IED Agents what condition should be achieved and wait the confirmation from IED Agent if it is done. If one or several Relay Agents failed to respond, the Coordination Agent will send the request for back-up planning list to the Configurator Agent and the new list of "Relay Configuration" will be issued to Coordination Agent. The "Relay Configuration" list is saved in the Database Agent. The whole information will be send to Evaluation Agent in the System Layer to evaluate. In the Evaluation Agent all information will be evaluated and reported to the operator. The proposed algorithm can be seen in the flowchart in Figure 4.
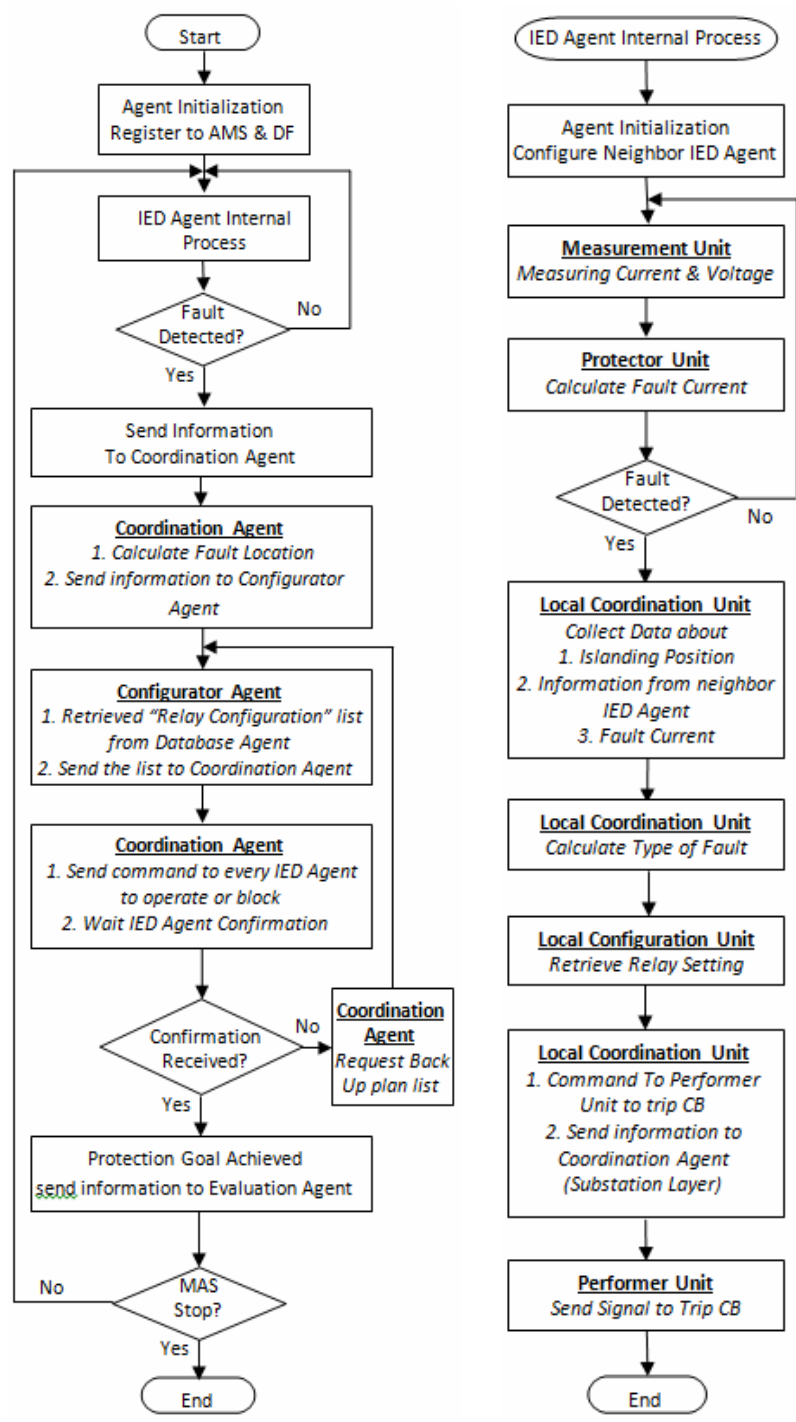

Figure 4. Flowchart of the proposed algorithm.

\section{Simulation and Results}

\subsection{Test System}

The proposed algorithm is simulated on the test system using Matlab-Simulink based on Figure 3. with threephase to ground fault occurred on F1 and F2. After initialization every agent will register the neighbor-agents to create protection zones (Table 1). If the fault current occurs, several agents will sense the fault current, first the agent will check the islanding status, and then the agent will compare the fault current to neighbor-agents and determine which relay setting should be chosen from the local database and trip the CB.

\subsection{Simulation Results}

The simulation result can be seen in Table 2. For the fault at F1, agent A02 and A06 will sense the same fault current so the agent will trip the $\mathrm{CB}$ at A02 and A06 to isolate the faulted line. When the fault at F2, Agent A06, A09-A12 sense the fault current and trip the corresponding CB.

\section{Conclusions}

The presence of DER in the distribution system will give many advantages to users and providers. In other hand DER penetration in the radial distribution system will change the power flow in the system and make the protection coordination become complicated. The possibility of islanding operation is show that the relay setting should be changed dynamically based on grid topology.

The small generator used in DER has limited fault current and small inertia. This makes easily to get "out of step" that means the protection should react fast. MAS' concepts are used to distribute the adaptive algorithm in equipment agents and reduce the operation time of the whole protection system.

Table 1. Neighbor-agents for some agents.

\begin{tabular}{ccccccc}
\hline Agent & A02 & A06 & A09 & A10 & A11 & A12 \\
\hline & A03 & A05 & A10 & A09 & A09 & A09 \\
$\begin{array}{c}\text { Neighbor- } \\
\text { Agents }\end{array}$ & A04 & --- & A11 & A11 & A10 & A10 \\
& A06 & --- & A12 & A12 & A12 & A11
\end{tabular}

Table 2. Simulation results.

\begin{tabular}{ccccccc}
\hline \multirow{2}{*}{ Fault } & \multicolumn{5}{c}{ Agents / CB Trip Time (milliseconds) } \\
\cline { 2 - 7 } & A02 & A06 & A09 & A10 & A11 & A12 \\
\hline F1 & 3.75 & 32.5 & NT & NT & NT & NT \\
F2 & NT & 4.55 & 9.45 & 15.05 & 15 & 15 \\
\hline
\end{tabular}

a. Other CB Agents are not trip.; b. NT means Not Trip. 
The Simulation results show that the algorithm can works selective and fast. The trip time is between 3.75 to 15.05 milliseconds.

\section{REFERENCES}

[1] R. C. Dugan and T. E. McDermott, "Distributed Generation," IEEE Industry Applications Magazine, Vol. 8, No. 2, 2002, pp. 19-25. doi:10.1109/2943.985677

[2] X. J. Zeng, et al., "Multi-agents Based Protection for Distributed Generation Systems," Electric Utility Deregulation, Restructuring and Power Technologies, 2004 (DRPT 2004), Proceedings of the 2004 IEEE International Conference on. Vol. 1. IEEE, 2004.

[3] A. R. Haron and V. Mohamed and V. Shareef, "A Review on Protection Schemes and Coordination Techniques in Microgrid System," Journal of Applied Sciences, Vol. 12, No. 2, 2012, pp. 101-112.doi:10.3923/jas.2012.101.112

[4] M. R. Islam, "Study of Micro Grid Safety \& Protection Strategies with Control System Infrastructures," Smart Grid and Renewable Energy, Vol. 03, No. 01, 2012, pp. 1-9, doi:10.4236/sgre.2012.31001

[5] E. Coster, J. Myrzik and W. Kling, "Effect of DG on Distribution Grid Protection," Distributed Generation, D $\mathrm{N}$ Gaonkar (Ed.), InTech, Available from: http://www.intechopen.com/books/distributed-generation/ effect-of-dg-on-distribution-grid-protection doi: $10.5772 / 8880$

[6] M. PazComech, Miguel Garcia-Gracia, Samuel Borroy and M.TeresaVillen (2010). Protection in Distributed Generation, Distributed Generation, D N Gaonkar (Ed.), ISBN: 978-953-307-046-9, InTech, DOI: 10.5772/8887. Available from:

http://www.intechopen.com/books/distributed-generation/ protection-in-distributed-generation doi: $10.5772 / 8887$

[7] I. Xyngi and M. Popov, "Smart Protection in Dutch Medium Voltage Distributed Generation Systems," Innovative Smart Grid Technologies Conference Europe (ISGT Europe), 2010 IEEE PES. IEEE, 2010.

[8] O. Alexandre, "Advanced Architectures and Control Concepts for MORE MICROGRIDS: Novel protection systems for Microgrids," Deliverable DC2, Nov (2009).

[9] S. D. J. McArthur, E. M. Davidson, V. M. Catterson, A. L. Dimeas, N. D. Hatziargyriou, F. Ponci and T. Funabashi, "Multi-Agent Systems for Power Engineering Applications - Part I : Concepts , Approaches, and Technical Challenges," Power Systems, IEEE Transactions on, Vol. 22, No. 4, 2007, pp. 1743-1752.
doi:10.1109/TPWRS.2007.908471

[10] S. D. J. McArthur, E. M. Davidson, V. M. Catterson, A. L. Dimeas, N. D. Hatziargyriou, F. Ponci and T. Funabashi, "Multi-Agent Systems for Power Engineering Applications - Part II : Technologies, Standards, and Tools for Building Multi-agent Systems," Power Systems, IEEE Transactions on, Vol. 22, No. 4, 2007, pp. 1753-1759. doi:10.1109/TPWRS.2007.908472

[11] S. D. J. McArthur and E. M. Davidson, "Concepts and Approaches in Multi-Agent Systems for Power Applications, (Invited Paper)," Proceedings of the 13th International Conference on, Intelligent Systems Application to Power Systems, 2005, pp. 391-395.

[12] M. Yang and Y. Zhu, "A Cooperative Protection System with Multi-Agent System," Conference and Exhibition: Asia and Pacific, 2005, pp. 1-4.

[13] Q. Zhao and S. Liu, "Relay Protection Based on Multi-Agent System," IEEE International Conference on Electrical and Control Engineering (ICECE), pp. 286-289.

[14] Y. M. Yu and Z. Y. Li, "Application of Multi-Agent System for Adaptive Protection System," Science paper Online, Available from: http://www.paper.edu.cn.

[15] J. H. Chen, S. Chen and Y. M. Yang, "Multi-agent Based Protection Relay System for Transmission Network," in Machine Learning and Cybernetics, 2003 International Conference on, Vol. 4, 2003, pp. 2251-2254.

[16] H. Gao, Q. Pang and Y. An, "New Type of Protection and Control Method for Smart Distribution Grid," 11th IET International Conference on Developments in Power Systems Protection (DPSP 2012), 2012, pp. 24-24.

[17] C. Fukui, "A Cooperative Protection System with an Agent Model," IEEE Transactions on Power Delivery, Vol. 13, No. 4, 1998, pp. 1060-1066. doi:10.1109/61.714454

[18] B. Stedall, P. Moore and A. Johns, "An Investigation into the Use of Adaptive Setting Techniques for Improved Distance Back-up Protection," Power Delivery, IEEE, Vol. 11, No. 2, 1996, pp. 757-762.doi:10.1109/61.489332

[19] A. A. Bittencourt, M. R. De Carvalho and J. G. R. M. Ieee, "Adaptive Strategies in Power Systems Protection using Artificial Intelligence Techniques," in 2009 15th International Conference on Intelligent System Applications to Power Systems, 2009, pp. 1- 6.

[20] P. C. Maiola and J. G. Rolim, "A Multi-Agent System for Protection Coordination of Radial Systems in the Presence of Distributed Generation," Systems Protection, 2012, pp. 131-131. 